\title{
Orientation selectivity of the McCollough effect: Analysis by equivalent contrast transformation
}

\author{
STEPHEN R. ELLIS \\ Brown University, Providence, Rhode Island 02912
}

\begin{abstract}
Using a color-cancellation technique, the strength of the McCollough effect was measured in units of excitation purity. The strength was studied both as a function of the contrast of the adapting gratings and as a function of the angle $X$ between the axes of the test and the adapting gratings. Results were well described as a linear function of the contrast of the adapting gratings and as a $\cos (2 \times)$ function of the angle. Both functions were combined to express an equivalent contrast transformation which converts the measurements of orientation tuning into a unit comparable to that used for other kinds of orientation-specific aftereffects. The orientation tuning was found to be very broad with a half-width at half amplitude of approximately $27^{\circ}$. This estimate is considered to be a substantial underestimate of the actual tuning of the aftereffect's substrate.
\end{abstract}

If a person views a vertical line grating of black and magenta bars alternated with a horizontal grating of black and green bars, his perception of black and white gratings is altered. Vertical gratings appear faintly green and horizontal gratings appear faintly magenta. The orientation specificity of this type of perception aftereffect, first described by McCollough (1965), has been one of its defining characteristics. Early determinations of its orientation tuning (Fidell, 1970; Hepler, 1971; Stromeyer \& Mansfield, 1970; Teft \& Clark, 1968) were hampered, however, either by the variability or the subjectivity of the measures of aftereffect strength that were used. Development of more objective and reliable measures of the strength of McCollough effects has made possible the reexamination of orientation tuning to determine its specific character (Riggs, White, \& Eimas, 1974; White, 1976). In addition, these techniques allow a systematic attempt to measure orientation specificity in units comparable to those used for measuring other types of aftereffects, allowing control for nonlinearities of measurement.

Measurement nonlinearities can significantly distort measurements of orientation specificity, as has been shown by Blakemore and Nachmias (1971). This distortion is particularly evident if the method of measuring aftereffect strength begins to saturate

A preliminary report of the research reported in the present paper was made at ARVO 75 by S. R. Ellis and Kevin Castellan. The author was supported by an N.I.H. postdoctoral traineeship from PHS Grant E700744 to Dr. Lorrin Riggs while conducting these investigations, and he is deeply indebted to Dr. Riggs, Dr. Keith White, and Kevin Castellan for intellectual and material assistance. The author's present address is: School of Optometry, University of California, Berkeley, California 94720. out for higher levels of aftereffect strength. Under these circumstances, unit changes in strong aftereffects produce variations in the index of measurement which are small in comparison to those produced by unit changes of much weaker aftereffects. Circumstances like these can lead to observations that may reflect the idiosyncratic relationship between the measurement technique and the aftereffect rather than properties of the aftereffect itself. An aftereffect, for example, might be described as having a broad orientation specificity, since the index of its strength does not change very much as the test stimuli used to observe it are rotated. Such an observation alone may be misleading, since the small change in the measured strength of the aftereffect quality could equally well be due to some insensitivity of the measurement technique at the particular strength of aftereffect used. This type of problem is particularly important for a variety of aftereffects, especially when neither a natural nor a conventional means of measuring the strength of the particular aftereffect has evolved, and is the organizing question of the following two experiments.

\section{EXPERIMENT 1}

\begin{abstract}
Method
Apparatus and Stimuli. The apparatus and stimuli used in the experiment were basically similar to those described by White (1976). Subjects alternately viewed colored orthogonal line gratings of 3 cycles/deg. Circular patterns used to produce the aftereffect were made with high-contrast Kodalith film and had a rectangular luminance profile with $65 \%$ clear area. They were projected by adjacent standard $35-\mathrm{mm}$ projectors onto a circular white diffusing screen subtending $9^{\circ}$ at a viewing distance of $1 \mathrm{~m}$. Alternation of the patterns was achieved by rotating a $180^{\circ}$
\end{abstract}




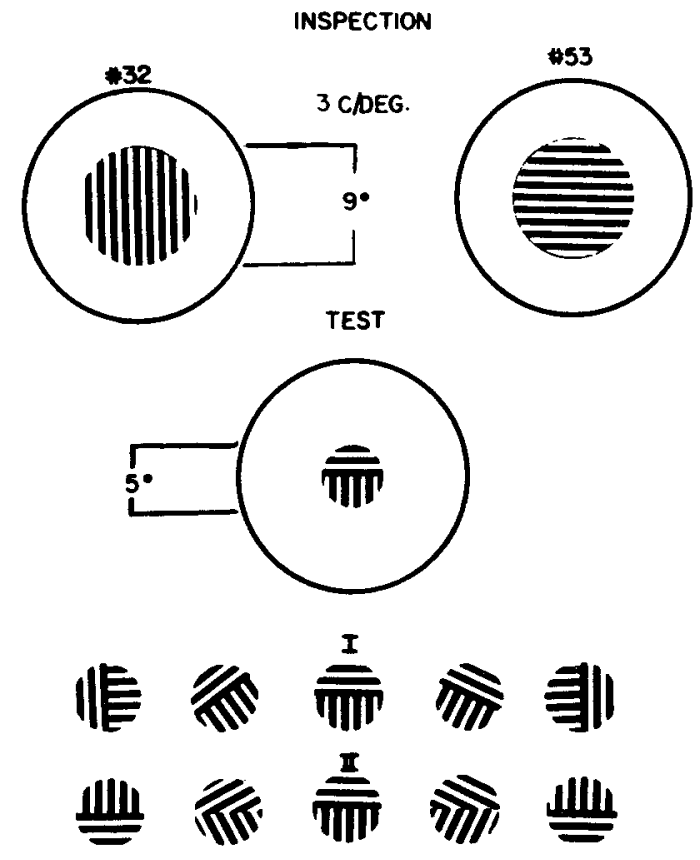

Figure 1. Schematic illustration of the structure of the stimulus fields used to produce and test McCollough effects.

sector disk in front of the pair of projectors. Each orthogonal grating was separately colored magenta or green by Kodak Wratten filters, No. 32 and No. 53, respectively. A SEI spot photometer and a UDT photodiode photometer were used to calibrate the luminance and contrast of the stimuli used. The complementary colored inspection patterns were presented centered in a white, circular surround of $29 \mathrm{ml}$ which was illuminated by four F8T5 cool-white flourescent lamps. The space-averaged luminance of the inspection gratings used to produce the aftereffect was kept in the range of $12 \pm 2 \mathrm{~mL}$. Previous results by White (1976) show that this range of variation would have negligible effects on the production of McCollough effects. Luminance contrast of the inspection stimuli took on six different values: $.46, .31, .19, .08, .04, .00 .^{\prime}$ The four lowest contrasts were produced with Kodak Tri-X film, while Kodalith Type III was used to produce the highest contrast grating. Space averaged luminance was adjusted with neutral filters attached to the films (Kodak Wratten No. 96).

The strength of the McCollough effect produced by inspection was measured using a null-match color-cancellation. technique identical to that described by White (1976). In brief, a variable chromaticity test pattern is produced by projecting a circular bipartite test pattern on a $5^{\circ}$ circular screen which replaced the $9^{\circ}$ screen used to present the inspection patterns. The top half contained a horizontal grating and the bottom half a vertical grating, both with the same luminance profile as the inspection gratings. The space-averaged luminance was approximately $15 \mathrm{~mL}$. Special optical arrangements previously described by White (1976) allowed the subject to adjust the chromaticity of the light used to project the test pattern, so it appeared with the desired saturation of magenta on top and green on bottom, or vice versa. Saturation was measured in terms of the average excitation purity $\left(\mathrm{P}_{\mathrm{e}}\right)$ of both colored regions. A sign convention was adopted so that positive excitation purity indicated magenta added to the top and green to the bottom. Negative indicated the reverse. Thus it was possible for the subject to adjust the objective coloration of the test pattern to cancel any subjective coloration seen. Such an adjustment is called a null match for color and occurs when the subject judges that the colors of both parts of the field are matched. In general, this neutral point also appeared achromatic, though some subjects reported it as slightly greenish relative to the white surround.

During all phases of the experiments, the subject's head was stabilized with a chinrest and head restraint which allowed him to view all stimuli monocularly through a $2-\mathrm{mm}$ artificial pupil for long periods of time, up to $2 \mathrm{~h}$. A dove prism was placed just beyond the artificial pupil to provide control of stimulus orientation in Experiment 2, but was in place in Experiment 1 to insure stimulus comparability. ${ }^{2}$

Subjects. Six paid subjects served in the experiment. Five were naive, unpracticed psychophysical observers and one was a practiced observer. All had vision corrected to at least 20/30, and all passed the color perception test on the Bauch and Lomb Orthorater.

Procedure. Before beginning the actual experiment, the subject was familiarized with the equipment and given practice making null matches while viewing the test pattern. His task was to adjust the color of both parts of the field after an objective mismatch had been produced by displacing the disk that controlled the coloration. This practice continued until the average of the subject's last 10 matches stabilized within $0.8 \mathrm{P}_{\mathrm{c}} \times 10^{-2}$ of an objective match. After this practice, the subject sat in the testing room for $5 \mathrm{~min}$ with only the surround light on while monocularly viewing different parts of the field seen through the artificial pupil. He then made 10 null matches which were averaged to provide a preinspection baseline. Aftereffect strength was measured as a deviation from this baseline.

Each of the six subjects had McCollough aftereffects produced on six occasions by separate 15 -min inspections of colored gratings with each of the six levels of contrast used. Following monocular, left-eye inspection, sets of 10 null matches were taken beginning 1 , $16,64,256$, and 1,024 minutes after the end of inspection. Sufficient time elapsed between each of the inspection sessions to allow previously established aftereffects to decay to levels not statistically significantly different from the previous baseline. One week was usually sufficient. The order of presentation of the contrast levels was randomized.

\section{Results}

Typical results for one subject from three different inspection sessions conducted with high (.46), medium (.19), and low (.04) contrast are shown in Figure 2. These results clearly show the aftereffect strength to be influenced by inspection pattern contrast. They also raise the question of how best to index the strength of any particular aftereffect, since $\Delta \mathrm{P}_{\mathrm{e}}$, the increase in saturation necessary to produce a null match after inspection, decreases with time (Riggs et al., 1974). By convention, one can take the estimated aftereffect strength at $1 \mathrm{~min}$ after the end of inspection as an index of the initial strength of aftereffect. As illustrated in Figure 2, this value is conveniently derived from a particular decay curve by fitting a regression of the form $\Delta \mathrm{P}_{\mathrm{e}}=\mathrm{a} \ln (\mathrm{t})+\mathrm{b}$ to the means of the five batches of null matches taken after inspection, where $t$ is time after end of inspection in minutes. Thus $b$, the aftereffect strength at 1 minute, i.e., when $\ln (t)=0$, is the index of aftereffect strength.

This index of aftereffect strength was determined for all inspection sessions. Typical results from three subjects are presented in Figure 3 as a function of 


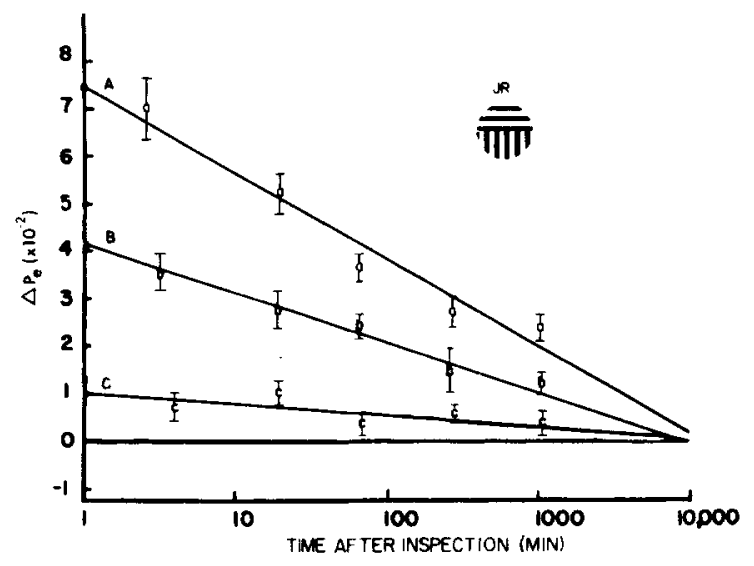

Figure 2. Graph illustrating the time course of the decay of three separate McCollough effects produced with three different levels of inspection contrast: $A .43, B .19$, and $C .04$. The data points are means of 10 null-match settings expressed as a deviation $\Delta P_{e}$ from a preinspection baseline. The error bars indicate \pm 1 standard error. The lines are plotted from regression used to calculate the indices of initial aftereffect strength. The somewhat unusual plot against log time is purely descriptive and is used here for computation convenience.

inspection pattern contrast. The approximately linear relationship evident is characteristic of our experimental results.

\section{Discussion}

These measures of aftereffect strength are a nearly linear function of inspection pattern contrast. This form of relationship distinguishes the McCollough

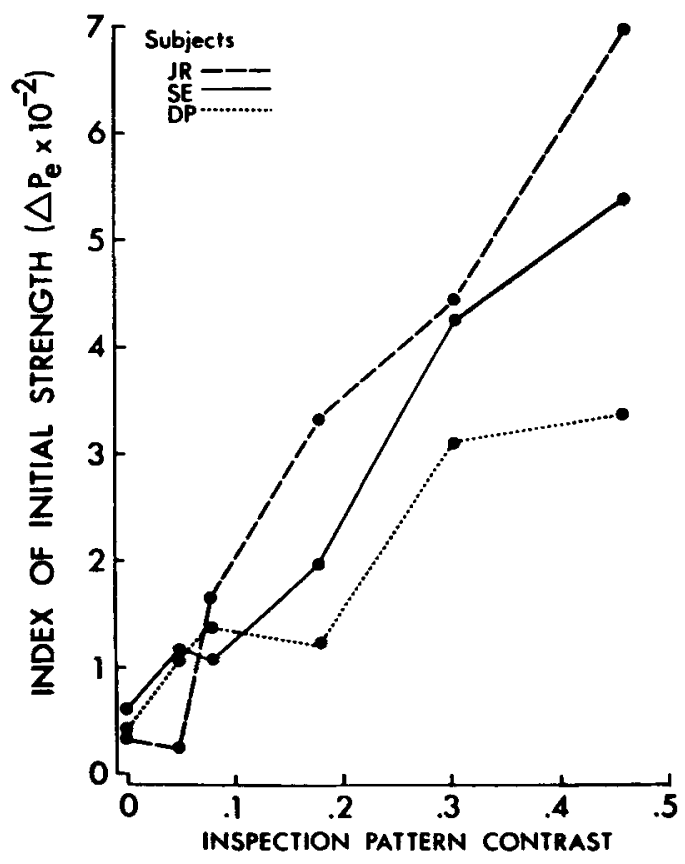

Figure 3. Indices of initial aftereffect strength for three subjects plotted as a function of inspection pattern contrast. effect from achromatic aftereffects which more typically vary as nonlinear functions of contrast (Blakemore \& Nachmias, 1971; Sharpe \& Tolhurst, 1973a, 1973b). Recent preliminary results by White (Note 1) confirm a nearly linear relationship between similar measures of aftereffect strength and test pattern contrast. The linear form of this functional relationship has significant importance to interaftereffect comparisons of orientation specificity.

\section{EXPERIMENT 2}

The orientation specificity of the McCollough effect was determined by measuring the decrease in aftereffect strength as the axes of the test pattern gratings were rotated relative to the inspection pattern. Two different methods of rotation were used and their results compared.

\section{Method}

Apparatus and Stimuli. The apparatus used in this experiment was identical to that of Experiment 1. The test pattern gratings were rotated by rotating the dove prism in the viewing channel either clockwise or counterclockwise. This technique (Method 1), thus rotated the entire test pattern, including the margin between the two halves of the bipartite field (see Figure 1). A second technique (Method 2) was used to check the first and to insure that whatever function was found did not depend upon a peculiarity of the way the grating bars were cut off by the bipartite field boundary. In this method, the orientation of the margin was kept constant while the upper and lower gratings were rotated by equal amounts. This was achieved by making up a series of test slides of the type illustrated in the lower part of Figure 1.

Subjects. Four of the six subjects previously tested in Experiment 1 returned after approximately 3 weeks for the first of two additional inspection sessions. Orientation specificity was determined using Method 1 on the first of these, and again after approximately 3 weeks, with Method 2 on the second.

Procedure. The inspection procedure used for orientation specificity experiments was identical to that used in Experiment 1. The testing procedure differed in that the aftereffect strength was measured repeatedly over a 90 -min period with test patterns rotated clockwise (CW) and counterclockwise (CCW) with respect to the inspection pattern. The order of presentation of test patterns was arranged to produce a sequence of orientations such

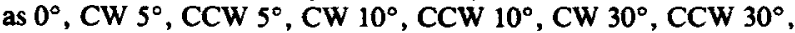
$\mathrm{CW} 45^{\circ}$, and $\mathrm{CCW} 45^{\circ}$. This sequence and its reverse were alternately used to direct the presentation of test patterns, first to obtain baselines for each orientation and later to obtain aftereffect measures. Ten repetitions of the sequence and its reverse, totaling 180 null matches, were used to obtain baselines for each orientation. After inspection, 25 to 30 repetitions of the sequence were used to measure aftereffect strength depending upon the speed with which the subject made null matches. This testing sequence allowed the measurement of aftereffect strength for the various test pattern orientations at many equally distributed points in time after inspection. The equal spacing of the individual test measurements allowed regression to be applied in the manner of Experiment 1 for the determination of an index of initial aftereffect strength at each test pattern orientation.

\section{Results}

Figure 4 illustrates the kind of orientation tuning function that is obtained for one subject, D.P. As usual, the aftereffect for each test pattern is expressed as a deviation from its preinspection base- 


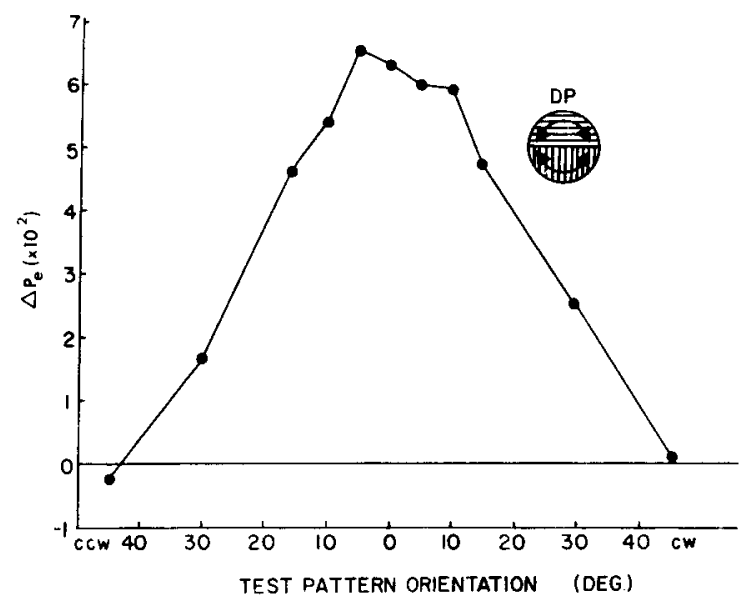

Figure 4. Representative orientation tuning function of one subject using Method 2 with test pattern organizations $0^{\circ}$, $\mathrm{CW} / \mathrm{CCW} 10^{\circ}, 15^{\circ}, 30^{\circ}, 45^{\circ}$. The ordinate is the index of initial aftereffect strength. Inspection pattern contrast was .43 .

line. The data in this figure were collected using rotation Method 2 and demonstrates the typically broad tuning found directly. Similar tuning functions for other subjects can be seen in the left halves of Figure 5 and 6 . In these figures, the tuning curves are collapsed across direction of rotation for comparison with the same subject's data from Experiment 1. In Figure 5, for example, we can see that the maximum aftereffect strength determined during the orientation tuning of an aftereffect produced by 15 -min viewing of inspection patterns with 0.31 contrast was about $4.8 \mathrm{P}_{\mathrm{e}} \times 10^{-2}$. This corresponds, as it should, to the strength of aftereffect for a contrast of .31 shown in the contrast function on the right. This close correspondence in regular data, like that of Figure 5, indicates that the somewhat different testing procedures used in Experiments 1 and 2 do not substantially alter measures of aftereffect strength. The less regular data in Figure 6 shows that such a close correspondence is not always achieved. Here the maximum of a tuning curve determined for an aftereffect produced with .43 contrast is about $20 \%$ greater than would be expected from the contrast curve on the right.

Attempts were made to find appropriate simple descriptions of the orientation tuning functions using regression based on several different models: $a x+b, a \log (x)+b, a x^{b}$, and $a \cos (2 x)+b$, where $\mathrm{x}$ is the angle in degrees between inspection and test grating. Using $r^{2}$ as a measure of goodness of fit, we found six of the eight tuning functions we measured to be best described by the $\cos (2 x)$ function, and the remaining two to be best fit by linear functions. Once a simple description of the tuning function is found, it is possible to quantify the sharpness of tuning by finding how the test pattern orientation must deviate from that of the inspection pattern to produce a $50 \%$ drop from the maximum aftereffect strength. This angle is called the half-width at half-amplitude, or half-width for short (Blakemore \& Nachmias, 1971). It is illustrated in a direct way in the left halves of Figures 5 and 6, which illustrate our most typical tuning results described by $\cos (2 \mathrm{x})$.

As indicated in the introduction, such directly determined orientation specificities may be distorted by nonlinearities of measurement. The equivalent

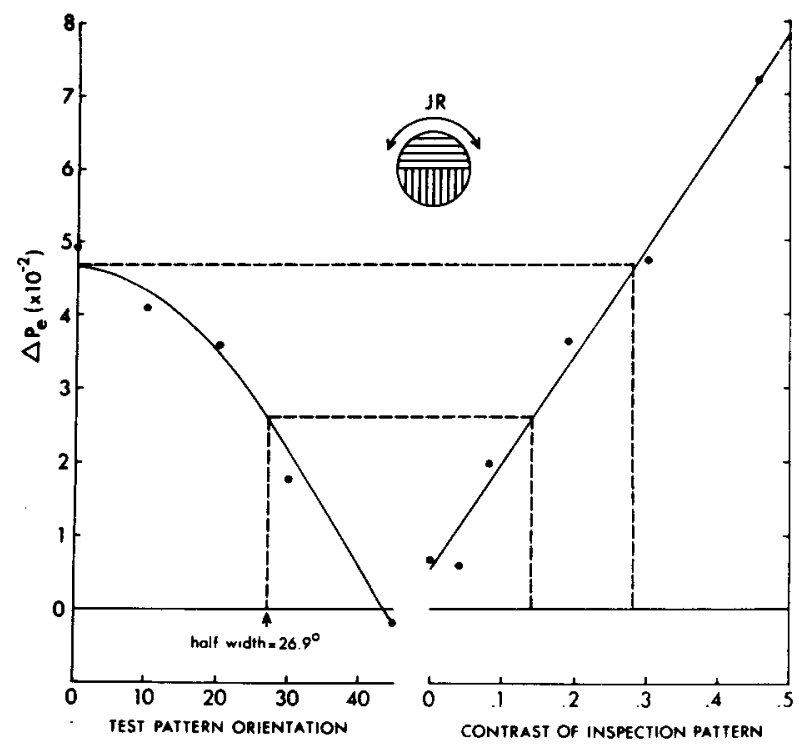

Figure 5. Orientation tuning and effect of inspection pattern contrast for subject J.R. in terms of index of initial aftereffect strength. Method 1 orientation luning was used and is coliapsed across direction of rotation. The left-hand curve represents the regression chosen as the best simple description of the orientation data, similarly for the regression line through the contrast data on the right panel. Inspection pattern contrast for the orientation tuning was. $\mathbf{4 3}$. The broken lines indicate the correspondence between the two graphs to determine the half-width in units of "equivalent contrast."

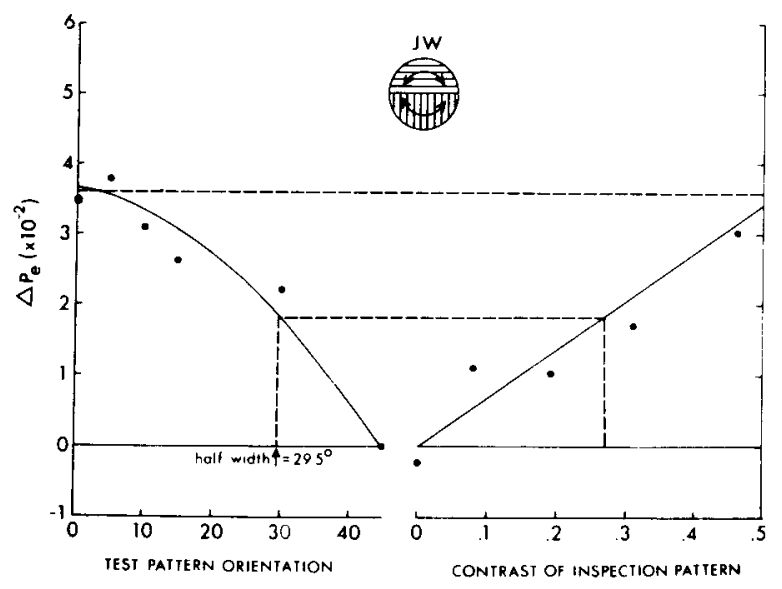

Figure 6. Orientation tuning and effect of inspection pattern contrast for subject J.W. in terms of index of initial aftereffect strength. Method 2, inspection pattern contrast for orientation tuning was $\mathbf{. 4 3}$. 
contrast transformation devised by Blakemore and Nachmias (1971) can remove these nonlinearities by providing an equivalent metric for the measurement of the strength of different aftereffects. The transformation is accomplished with respect to orientation constancy as follows: For every point in the original tuning curve find the inspection pattern contrast that would be necessary to produce an equivalent aftereffect strength. These "equivalent contrasts" then are used as a transformed measure of aftereffect strength for replotting of the tuning function and a new determination of half-width. ${ }^{3}$

Transformed half-widths can be found, however, without plotting the entire transformed function: For each subject, find the contrast level in his contrast function that corresponds to the maximum aftereffect strength in the paired untransformed tuning function. Divide this equivalent contrast by two and work backwards from the contrast function to the tuning function to find which test pattern orientation corresponds to this halved "equivalent contrast." The results of this procedure are illustrated by the broken lines linking the tuning and contrast curves in Figures 5 and 6.

Equivalent contrast transformations have been carried out for eight tuning functions we have determined. The transformed functions are presented in Table 1 along with respective half-widths and $r^{2}$. Since all the contrast functions were simply described best by linear functions, these latter two values were unaffected by the transformation. Thus, the results of Experiment 1 interact with those of Experiment 2 to support the claim that the orientation tuning function of the McCollough effect is unusually broad, with half-widths clustering around $27^{\circ}$. This feature would distinguish the effect from achromatic aftereffects which for comparable spatial parameters have tuning curves for half-widths typically less than $15^{\circ}$ (Sharpe \& Tolhurst, 1973a, 1973b). Furthermore, the result serves to set a psychophysical property to guide the search for a neurophysiological substrate.

\section{GENERAL DISCUSSION}

Further consideration of the manner in which the orientation data were collected indicates that the relatively broad tuning reported here is an underestimate of the actual tuning functions of the effect's substrate. Consider how, under the conditions used, rotation of the test pattern pits the magenta and greenish components of the aftereffect against each other. As the part of the test pattern which appears magenta is rotated, the color due to the aftereffect is lost not only because the test pattern orientation diverges from that of the inspection pattern producing the magenta aftereffect, but also because at the same time it approaches the orientation of the inspection pattern producing the greenish aftereffect. Thus,
Table 1

Chosen Simple Descriptive Orientation Tuning Functions Transformed to Relate Orientation in Degrees to Equivalent Contrast

\begin{tabular}{llcc}
\hline Subject & \multicolumn{1}{c}{$\begin{array}{c}\text { Transformed Tuning } \\
\text { Equation }\end{array}$} & $\begin{array}{c}\text { Half-Width } \\
\text { (Degrees) }\end{array}$ & $\mathbf{r}^{2}$ \\
\hline \multicolumn{4}{c}{ Method 1 } \\
S.R.E. & $\mathrm{C}_{\mathrm{e}}=.38 \operatorname{COS}(2 \mathrm{X})+.01$ & 29.5 & .96 \\
J.R. & $\mathrm{C}_{\mathrm{e}}=.34 \operatorname{COS}(2 \mathrm{X})+.06$ & 26.9 & .97 \\
J.W. & $\mathrm{C}_{\mathrm{e}}=.0088 \mathrm{X}+.29$ & 19.0 & .96 \\
D.P. & $\mathrm{C}_{\mathrm{e}}=.46 \operatorname{COS}(2 \mathrm{X})-.19$ & 25.5 & .98 \\
\multicolumn{1}{c}{ Method 2} \\
S.R.E. & $\mathrm{C}_{\mathrm{e}}=.0076 \mathrm{X}+.42$ & \\
J.R. & $\mathrm{C}_{\mathrm{e}}=.43 \operatorname{COS}(2 \mathrm{X})-.07$ & 26.5 & .98 \\
J.W. & $\mathrm{C}_{\mathrm{e}}=.49 \operatorname{COS}(2 \mathrm{X})-.02$ & 29.5 & .98 \\
D.P. & $\mathrm{C}_{\mathrm{e}}=.92 \operatorname{CoS}(2 \mathrm{X})-.10$ & 28.2 & .96 \\
\hline
\end{tabular}

Note-The $r^{2}$ are the corresponding coefficients of determination from the regression used to simply describe the orientation tuning, $d f=3$.

as the test pattern is rotated, the net aftereffect colors seen on each part are actually the resultant of the superposition of two approximately complementary aftereffect colors.

The effects of the superposition of such complementary aftereffect colors has fortunately been investigated by White (1976b), and has been shown to be simply additive: The excitation purity needed to cancel an aftereffect due to the superposition of two complementary aftereffects produced by separate inspections is the algebraic sum of the excitation purities necessary to cancel the aftereffects when produced in isolation (see discussion of aftereffect sign convention in Experiment 1). Accordingly, the effect of the double action of the rotated test pattern discussed above is to sharpen the measured tuning, driving it towards zero faster than would be the case were the components of the aftereffects being assessed in isolation.

This property of algebraic addition of complementary aftereffects suggests a possible way the orientation tuning functions reported above may be decomposed into their two complementary components. On the assumption that the $\cos (2 x)$ function gives, in general, the best description of the tuning; the trigonometric identity

$$
\cos (2 x)=\cos ^{2}(x)-\cos ^{2}(x-90)
$$

leads to the expectation that each of the aftereffect's orthogonal components would have very broad orientation tuning functions described by $\cos ^{2} x$.

We have conducted a preliminary investigation regarding this expectation using a horizontal green grating and a homogeneous magenta field matched for space-averaged luminance as the stimuli to be inspected. A collection of bipartite test patterns were prepared with variously oriented gratings in the lower half and a homogeneous field above as indicated in 


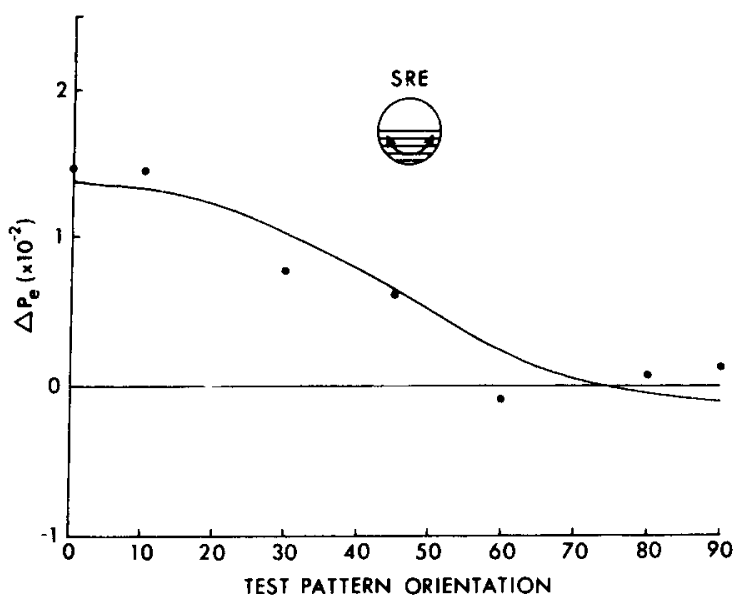

Figure 7. Orientation tuning curves for aftereffect produced by inspection of a horizontal green grating alternated with magenta homogeneous field collapsed across direction of rotation- $\Delta \mathbf{P}_{\mathrm{e}}\left(\mathbf{x 1 0 ^ { - 2 }}\right)=11.46 \cos (\mathrm{x})-.681 \mathrm{r}^{2}=.88$.

the inset to Figure 7. Thus, orientation tuning of only "half" a McCollough effect could be tested by presenting a subject with various test patterns akin to Method 2. Modifications in testing procedures resulted in a change in the visual appearance of the test stimulus when the subject equalized the chromaticity of both parts of the test pattern. ${ }^{4}$ Nevertheless, in spite of the perceptual differences in the method of measurement, the tuning function shown in Figure 7 is roughly as expected. Regardless of the specific shape of this function, it serves to provide support for the supposition that the underlying components of the McCollough effect are themselves very broadly tuned when examined in isolation by a method providing an unconstrained measure of tuning. The breadth indicated here, with half-width on the order of $45^{\circ}$, is such as to rekindle suspicion that physiological mechanisms with the properties of Harris and Gibson's (1968) "dipoles" may prove adequate to subserve the aftereffect. Such mechanisms, while not exhibiting orientation selectivity in a classical sense, nevertheless would be differentially activated by lines of different orientation. Populations of such units would be adequate to subserve a broad orientation tuning.

\section{REFERENCE NOTE}

1. White, K. D. Unpublished observations, 1977. (Available from Department of Psychology, University of Florida, Gainesville, Florida 32611.)

\section{REFERENCES}

Blakemore, C., \& Nachmias, J. The orientation specificity of two visual aftereffects. Journal of Physiology, 1971, 213, 157-174.

FIDELL, L. S. Orientation specificity in chromatic adaptation of human edge detectors. Perception \& Psychophysics, 1970, 8, 235-237.

HARRIs, C. S., \& Gibson, A. R. Is orientation-specific color adaptation in human vision due to edge-detectors, afterimages, or "dipoles?" Science, 1968, 162, 1506-1507.

HEPLER, N. K. Motion-contingent color aftereffects: $A$ lasting modification of perception. Unpublished PhD dissertation, McGill University, March 1971.

McCollough, C. Color adaptation of edge detectors in the human visual system. Science, 1965, 149, $1105-1106$.

Riggs, L. A., White, K. D., \& Eimas, P. D. Establishment and decay of orientation contingent aftereffects of color. Perception \& Psychophysics, 1974, 16, 535-542.

Sharpe, C. R., \& Tolmurst, D. J. The effects of temporal modulation of the orientation channels of the human visual system. Perception. 1973, 2, 23-29. (a)

Sharpe, C. R., \& Tolhurst, D. J. Orientation and spatial frequency channels in peripheral vision. Vision Research, 1973, 13, 2103-2112. (b)

Stromeyer, C. F., III, \& Mansfield, J. W. Colored aftereffects induced with moving edges. Perception \& Psychophysics, 1970. 7. 108-114.

Teft, L. W.. \& Clark, F. The effect of stimulus density on orientation specific aftereffects of color adaptation. Psychonomic Science, 1968, 11, 265-266.

WhITE. K. D. Luminance as a parameter in the development and testing of McCollough effects. Vision Research, 1976, 15, 297-302. (a)

WhITE, K. D. Studies of orientation-contingent color aftereffects. Unpublished PhD dissertation, Brown University, 1976. (b)

\section{NOTES}

1. Contrast, $\mathrm{c}$, was defined according to the following formula:

$$
c=\left(\mathrm{L}_{\max }-\mathrm{L}_{\min }\right) /\left(2 \mathrm{~L}_{\mathrm{avg}}\right)
$$

where $\mathrm{L}_{\max }, \mathrm{L}_{\min }$, and $\mathrm{L}_{\text {avg }}$ denote maximum minimum, and space-averaged luminance, respectively.

2 . Since the dove prism in the viewing channel was not normal to the viewing screens and since the reflected light from the screen was partly polarized, optical rotation of the test grating in Method 1 resulted in about $5 \%$ change in spatial frequency and $10 \%$ change in contrast. These changes are negligible with respect to the experimental manipulations.

3. The equivalent-contrast transformation can be achieved algebraically where $y=f(c)$ represents the best simple functional description of the aftereffect strength as a function of inspection pattern contrast and $y=g(x)$ the best simple functional description of the aftereffect orientation tuning. The transformation yields an orientation tuning function in terms of equivalent contrast: $C_{e}=f^{-1}[g(x)]$.

4. The test patterns in Experiments 1 and 2 could be adjusted so that the color of both the upper and lower parts of the field were equivalent and approximately achromatic. Test patterns used in this last pilot experiment could not be similarly adjusted, since only the lower part of the field contained a pattern which would produce an aftereffect. Accordingly, the magenta and green complementary light added to the lower and upper parts of the field, respectively, would only cancel an aftereffect color in the lower part. Thus, as the magenta colored aftereffect in the lower field was cancelled by the addition of greenish light, magenta colored light was simultaneously added to the upper portion of the field. This double action of the cancellation light results in lower excitation purity of cancellation light necessary to achieve acceptable equality of chromaticity between the two parts of the test field: a match between the test field parts no longer requires complete cancellation of the aftereffect color. It is merely necessary to partially cancel it until it matches the magenta of the upper part of the field. In practice, these matches were harder to make than those used in Experiments 1 and 2.

(Received for publication March 31, 1977; revision accepted August 15, 1977.) 